Peter J. Strouse

Michael A. DiPietro

Fermin Saez

\section{Transient small-bowel intussusception in children on CT}

abnormality. Follow-up CT $[n=13$ (6 within $24 \mathrm{~h})$ ], ultrasound $(n=3)$, small-bowel follow-through $(n=4)$ and surgery $(n=3)$ showed no intussusception. In four patients with persistent symptoms, underlying pathology was identified requiring treatment (giardiasis, 2; small-bowel inflammation/strictures, 1; abscess and partial smallbowel obstruction after perforated appendicitis, 1). In 21 other patients, direct correlation of symptoms to CT abnormality was absent or questionable, no treatment was required, and there was no clinical or imaging evidence of persistence or recurrence. Conclusion: Most small-bowel intussusceptions identified in children by CT are transient and of no clinical significance.

Keywords Intussusception . Children · Small intestine Computed tomography

\section{Introduction}

Intussusception is a common gastrointestinal emergency in the pediatric population. Most diagnosed intussusceptions are ileocolic. Intrinsic small-bowel (enteroenteric) intussusceptions are much less frequently diagnosed. The purpose of this study was to determine the frequency and significance of small-bowel intussusceptions diagnosed in children by computed tomography (CT).

\section{Materials and methods}

Institutional review board approval was obtained for this study. Abdominal CT reports from July 1995 to April 2002 (approximately 6,000 studies) were reviewed to identify pediatric patients with possible small-bowel intussusception on CT. All studies with possible small-bowel abnormality by report were directly reviewed. In addition to the $\mathrm{CT}$, subsequent imaging studies pertinent to the abnormality (CT, sonography, small-bowel follow-through, enteroclysis) were reviewed. Medical records were reviewed to 
determine the indication for $\mathrm{CT}$, whether abdominal pain was a symptom at the time of CT, alternative diagnoses for the abdominal pain, underlying diagnoses predisposing to small-bowel intussusception, and treatment rendered for the abnormality or any underlying diagnoses.

A small-bowel intussusception was identified as an intraluminal mass within small bowel having a characteristic layered appearance and/or continuity of fat within the mass with adjacent mesenteric fat. Location within small bowel was determined by analysis of the appearance (size, fold pattern) and location of the bowel loop containing the mass.

\section{Results}

Twenty-five pediatric patients (16 male, 9 female) were identified with small-bowel intussusception on CT. Patient age ranged from 2 to 20 years, with a mean age of 11.2 years and a median age of 10 years. Two patients over 18 were included, one with lymphoma and the other with cystic fibrosis, both still under the care of our pediatric clinical colleagues. As we read approximately 6,000 pediatric abdominal CTs over the study period, the prevalence of small-bowel intussusception on CT at our institution is approximately $0.4 \%$. Twenty patients were scanned on single detector helical scanners, three on multidetector scanners and two on older "slide and shoot" scanners. Indications for CT were: pain, 12 (including 3 with vomiting and 5 with fever); trauma, 5 (4 with pain; 1 with pain status unknown); sepsis, 3; lymphoma, 3; follow-up of perforated appendicitis, 1 (with pain); premature adrenarche, 1 . In total, 17 patients had abdominal pain at or near the time of CT, 4 did not, and in the other 4 neurologic status inhibited evaluation for pain. Seven patients with pain had an alternative etiology for the pain identified on CT-pyelonephritis, splenic laceration, appendicitis, residual periappendiceal abscess with partial small-bowel obstruction, adnexal cyst, liver contusion/abdominal wall contusion, and ureteral calculus.

Twenty-three patients had one small-bowel intussusception. One patient had two simultaneous small-bowel intussusceptions and one patient had three. CT findings of intussusceptions were (Figs. 1, 2, 3, 4): intraluminal mass, 28/28 (100\%); layered appearance of mass, 19/28 $(68 \%)$; fat within the mass, $19 / 28(68 \%)$; continuity between fat within mass and mesenteric fat, 10/28 $(36 \%)$; mesenteric blood vessels extending into or to the mass, $9 / 28(32 \%)$; bowel dilatation proximal to mass, $2 / 28$ ( $7 \%$, both with underlying pathology). Short axis diameter of the intussusceptions ranged from 1.5 to $3.0 \mathrm{~cm}$ (mean $2.1 \mathrm{~cm}$ ). Cephalocaudad length of the intussusception ranged from 1.0 to $5.0 \mathrm{~cm}$ (mean $2.2 \mathrm{~cm}$ ). Oral contrast was seen within the mass on CT on at least one image in 8 of $28(29 \%)$ intussusceptions. Oral contrast was seen distal to the intussusception in 26 of 28 $(93 \%)$ instances. In the other 2 patients oral contrast had not yet reached the intussusception. Approximate
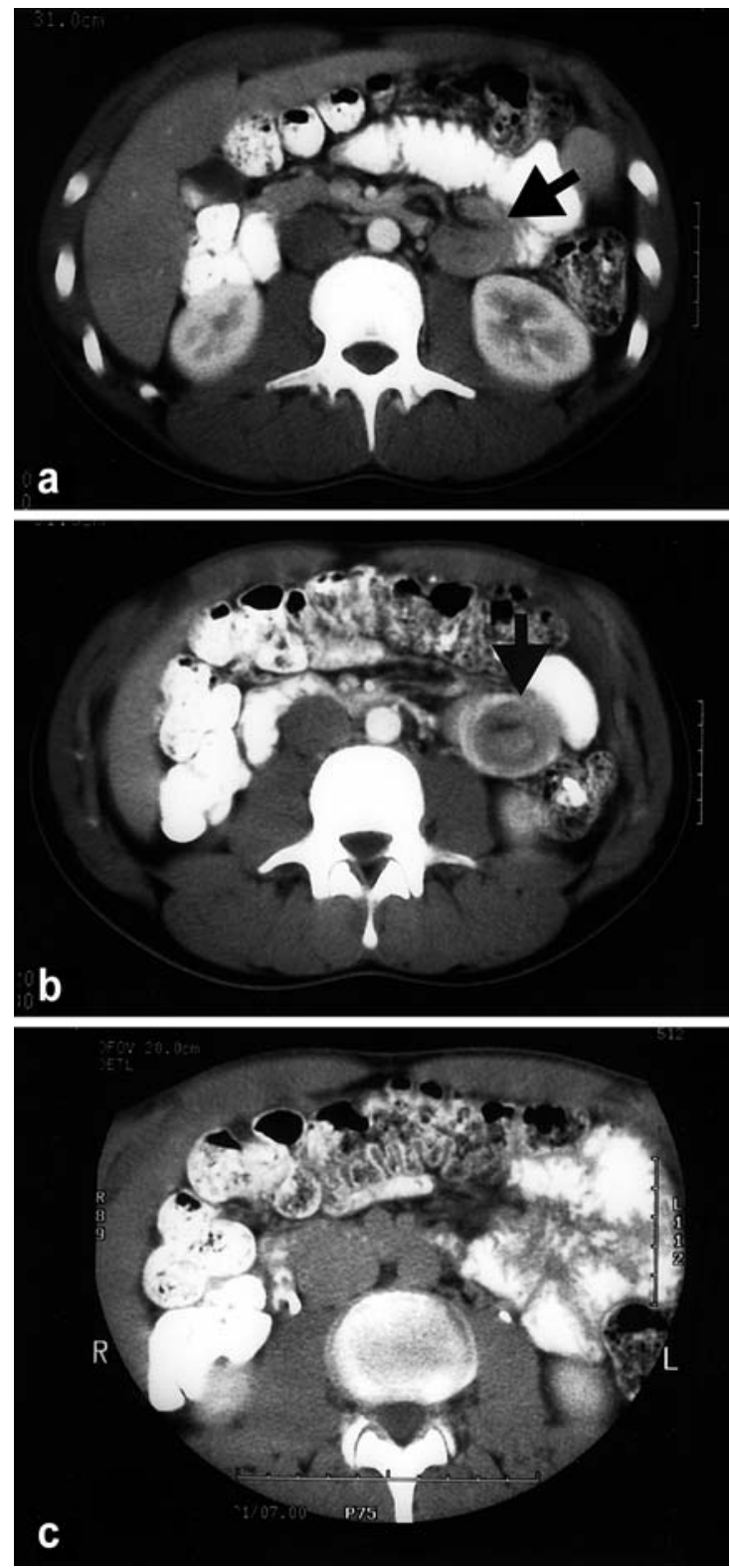

Fig. 1a-c Fifteen-year-old boy with MALT (mucosa-associated lymphoid tissue) lymphoma of the eyes. He had no abdominal symptoms. a, b Ten-millimeter-thick images show a small-bowel intussusception (arrows) in the left mid-abdomen. Mesenteric fat and a blood vessel are seen entering the intussusception (a). The intussusception has a layered ("target") appearance (b). c Limited 7-mm-thick repeat images 13 min later show resolution of the intussusception

location of intussusceptions as gauged by $\mathrm{CT}$ were: 15 jejunum $(54 \%), 5$ mid-small bowel $(18 \%$; = not sure if jejunum or ileum) and 8 ileum $(29 \%)$.

None of the intussusceptions appeared by CT to contain a lesion that could represent a lead point; however, in six patients abnormalities were seen that may have contributed to development of the intussusception. 

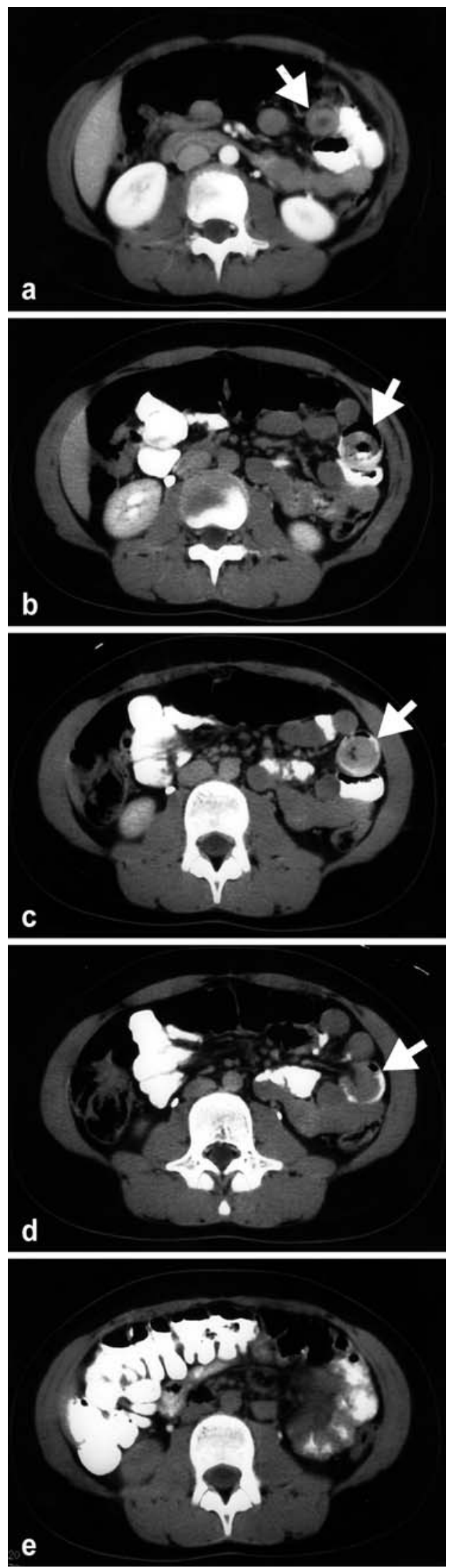

4

Fig. 2a-e Nine-year-old girl with abdominal pain after a fall out of a bunkbed. a An initial 7-mm-thick image shows a round softtissue opacity within jejunum (arrow). Slightly lower attenuation is seen centrally, and a crescent of low attenuation is seen at the anterior aspect of the lesion. No abnormality was seen on adjacent images. b-d Limited 3-mm-thick repeat images 13 min later show progression of the abnormality. An intraluminal mass is seen (arrows). Oral contrast material is seen within the mass at one location (b) and fat within the mass elsewhere (c, d). A crescent of gas is seen anteriorly between the intussusceptum and intussuscipiens. Continuity is noted between fat within the mass, and mesenteric fat and a mesenteric blood vessel is seen entering the mass (d). e Limited 5-mm-thick repeat images $4 \mathrm{~h}$ later show resolution of the abnormality. A small-bowel follow-through examination performed 2 days later was negative

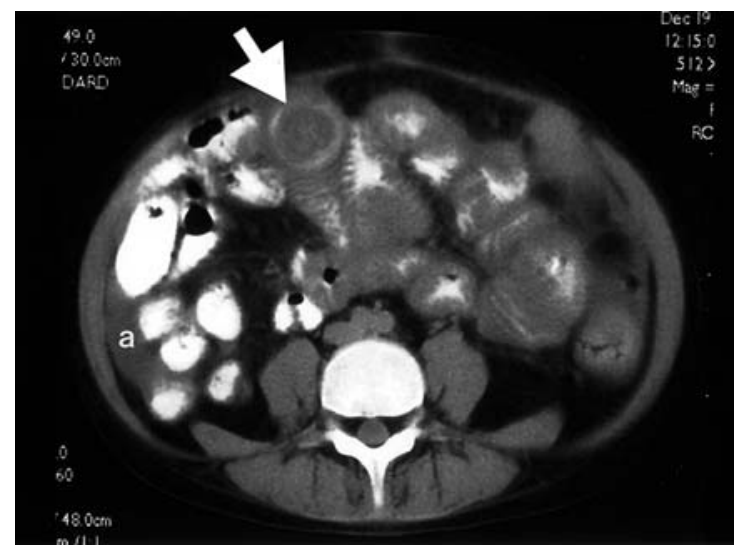

Fig. 3 Sixteen-year-old boy, status post liver transplant with sepsis, liver failure, and renal failure. The CT was performed without intravenous contrast. A small-bowel intraluminal mass is seen in the anterior right abdomen (arrow). The abnormality was seen on three adjacent images ( $7 \mathrm{~mm}$ thick) as well. There is diffuse small-bowel fold thickening and a small amount of ascites $(a)$

In three patients some adjacent small-bowel fold thickening was evident. Each of these patients was considered to be septic at the time of CT, and each had multiorgan dysfunction, including liver dysfunction. One of the three also had an underlying diagnosis of cystic fibrosis. All three of these patients survived the septic episode. One other patient had proximal small-bowel wall thickening, displaced from three sites of intussusception, and subsequently was proved to have giardiasis. One patient with known perforated appendicitis appeared to have a partial small-bowel obstruction at or near the site of intussusception. On immediate rescans, the intussusception resolved, but other findings of partial obstruction did not. Lastly, a 15-year-old girl with a lengthy transient intussusception with proximal dilatation proved to have three areas of small-bowel stricture subsequently identified on enteroclysis and resected at surgery. No intussusception was present at the time of surgery. Non-specific inflammation was seen on pathological examination of the resected small bowel. This 

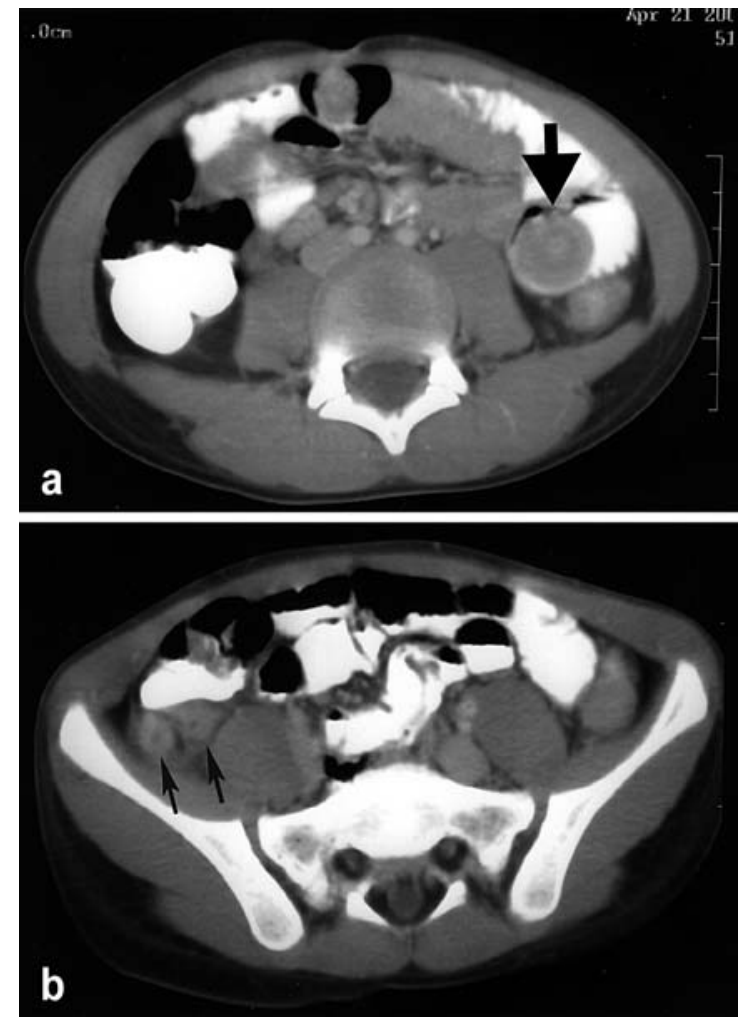

Fig. 4a, b Six-year-old boy with right lower quadrant abdominal pain and fever. a A small-bowel intussusception is seen in the left abdomen (arrow). The intussusception has a layered ("target") appearance. The intussusception was seen on three adjacent images ( $7 \mathrm{~mm}$ thick) as well. b An enlarged appendix (arrow) is seen with inflammatory changes in the adjacent fat. Limited repeat images 13 min later showed resolution of the intussusception. At surgery nonperforated appendicitis was confirmed, and no intussusception was seen

patient had previous documentation of small-bowel intussusceptions observed at the time of genitourinary tract surgery.

In addition to the aforementioned patients, a 2-yearold boy subsequently proved to have giardiasis, which likely predisposed him to small-bowel intussusception and warranted treatment. In 18 patients, no CT abnormality or subsequent diagnosis was evident to predispose the patient to small-bowel intussusception.

No patient had a persistent small-bowel intussusception requiring surgery. Apart from the four patients with underlying pathology identified, direct correlation of patient symptoms to the CT abnormality was absent or questionable. None of these other 21 patients required treatment for the intussusception and none had clinical or imaging evidence of recurrence or persistence of the intussusception. Fourteen patients had limited immediate repeat CT images through the area of abnormality. The abnormality had resolved in ten patients and persisted in four, increasing in size in one. Of the four patients with persistent abnormality on immediate rescans, two had scans approximately $4 \mathrm{~h}$ later, showing resolution of the abnormality, and one other had a small-bowel follow-through immediately after CT, showing no intussusception. In retrospect, the smallbowel study may have shown slight thickening of jejunal folds - this was one of the two patients subsequently confirmed to have giardiasis by laboratory examination of feces. The other patient with persistent abnormality on immediate rescanning is the 10-year-old girl, also subsequently diagnosed with giardiasis. She had two small-bowel intussusceptions on initial scans, which persisted with a third, new, intussusception on rescanning. Three other patients had small-bowel followthrough performed within 3 days of CT, all normal. Nine patients had subsequent CTs (time intervals: 1, 1, $5,7,7,12,30,51,90$ days), showing no intussusception and no small-bowel abnormality. Three patients had ultrasound examination. In one, the ultrasound was performed in the CT suite after marking the skin over the intussusception - no intussusception was found-immediate repeat CT images confirmed resolution. The other two ultrasounds were performed 2 and 5 days after $\mathrm{CT}$ and were normal. Two patients underwent laparoscopy, one which was normal and the other confirmed appendicitis, but no intussusception. In two patients transferred from elsewhere to our health care system, barium enemas had been performed as the intussusception had not been properly recognized as being in the small bowel. These enemas were negative. At least two additional enemas were avoided by our recognition of the small-bowel location of the intussusception.

\section{Discussion}

Small-bowl intussusceptions in children are uncommon. Pathologic entities that might predispose a child to small-bowel intussusception include infection, polyps, lymphoma, malabsorption syndromes, Meckel's diverticulum, duplication, cystic fibrosis, intramural hematoma, foreign body, and adhesions. Small-bowel intussusception has been reported to occur after abdominal surgery [1]. Small-bowel intussusception may also be idiopathic. Only four of our patients had definitive identification of an underlying disease process as causative of small-bowel intussusception. Three others had sepsis with multisystem disease (one of these also with cystic fibrosis) had small-bowel fold thickening evident on CT, potentially causally related to the development of small-bowel intussusception. However, 18 patients had no imaging, pathologic, or clinical confirmation of a cause for them to have intussusception. It is very likely that some of our patients had underlying pathology that was never recognized, but still led to the 
development of intussusception. This might include intramural hematoma in trauma patients, otherwise occult lymphomatous involvement of bowel and adhesions. If present, such abnormalities in themselves proved insignificant, as none of these 18 patients was proven to have recurrent intussusception or required specific treatment of a small-bowel abnormality.

This study is limited by its retrospective method. Additional information in regard to clinical symptoms and sonographic correlation might have been better addressed with a prospective study. This study is also limited by lack of pathologic correlation. CT findings were not corroborated with surgery. Because of the transient nature of the intussusceptions and lack of associated symptoms, surgical intervention was not warranted.

The findings of small-bowel intussusception on CT have been previously described $[2,3,4,5,6]$. The findings in our patients were similar. Small-bowel intussusceptions are seen as small (approximately $2 \mathrm{~cm}$ ) intraluminal masses. CT findings of a layered or "target" appearance of the mass, fat within the mass, particularly if in continuity with mesenteric fat, and mesenteric vessels entering the mass are features suggestive of intussusception. A crescent of gas or fluid may separate the mass (the intussusceptum) from the adjacent small-bowel wall (the intussuscipiens). The CT findings correlate with findings previously described for sonography [7, 8]. Specifically, an intraluminal mass with a layered appearance is seen on sonography. A crescent-shaped hyperechoic area on sonography represents invaginated mesenteric fat, which is seen as low attenuation on CT. The phenomenon of transient smallbowel intussusceptions on CT has been previously recognized in adults; however, knowledge of this is not widespread, particularly in children [2]. As a consequence, the significance of a small-bowel intussusception on CT may be clinically exaggerated. Transient smallbowel intussusception in children has been previously well documented by sonography in studies by Robben et al. [7] and Kornecki et al. [8]. Included in the study by
Kornecki et al. [8] were four patients identified with transient small-bowel intussusception by CT.

It is important to identify the location of the intussusception properly by the appearance and location of the involved bowel. In our experience, small-bowel intussusceptions are also much smaller than ileocolic intussusceptions on CT. By properly recognizing a smallbowel location, unnecessary enemas can be avoided. Limited, focused, immediate rescans on CT will often serve to confirm the transient nature of the abnormality expediently and definitively. Obviously, additional radiation dose is incurred, albeit small. Particularly in the asymptomatic patient, our results suggest that rescans may not be necessary. If persistence of the intussusception is an issue, sonography can also be utilized to document the presence or absence of an intussusception, using the preceding $\mathrm{CT}$ as a guide to the location.

From the literature and by our experience it is clear that some small-bowel intussusceptions can be clinically significant. They can persist and may require surgery or they may be the harbinger of underlying disease $[3,5,6]$. Patients with persistent, recurrent, or multiple smallbowel intussusceptions and patients with persistent symptoms therefore warrant further evaluation. Depending on the clinical situation, such evaluation might include repeat CT, ultrasound, or small-bowelstudy (follow-through or enteroclysis). Unless the intussusception is shown to be gone, it must be considered a possible cause of the patient's symptoms. In patients with recurrent symptoms and no other diagnosis, it is possible that an underlying disease that led to the small-bowel intussusception is the cause of symptoms via recurrent intussusception or other means. Given the complete absence of symptoms in some of our patients and the identification of alternative diagnoses in others, surely some, if not most, of the intussusceptions in this series were truly idiopathic. Our results indicate that the majority of small-bowel intussusceptions identified in children on CT are transient and of little or no clinical significance.

\section{References}

1. Kaste C, Wilimas J, Rao BN (1995) Postoperative small-bowel intussusception in children with cancer. Pediatr Radiol 25:21-23

2. Catalano O (1997) Transient small bowel intussusception: CT findings in adults. Br J Radiol 70:805-808

3. Merine D, Fishman EK, Jones B, et al (1987) Enteroenteric intussusception: CT findings in nine patients. AJR 148:1129-1132
4. Curcio CM, Feinstein RS, Humphrey RL, et al (1982) Computed tomography of entero-enteric intussusception. JCAT 6:969-974

5. Knowles MC, Fishman EK, Kuhlman JE, et al (1989) Transient intussusception in Crohn disease: CT evaluation. Radiology 170:814

6. Cox TD, Winters WD, Weinberger E (1996) CT of intussusception in the pediatric patient: diagnosis and pitfalls. Pediatr Radiol 26:26-32
7. Robben SG, Maertzdorf M, vanNuenen J, et al (1998) Asymptomatic small bowel intussusceptions in children (abstract). Pediatr Radiol 28:363-364

8. Kornecki A, Daneman A, Navarro O, et al (2000) Spontaneous reduction of intussusception: clinical spectrum, management and outcome. Pediatr Radiol 30:58-63 